\title{
The Structure of Strontium Titanate Bi-crystal Grain Boundaries
}

\author{
S. Dey,* J. P. Buban, ${ }^{*}$ and N. D. Browning*, ***, **** \\ * Chemical Engineering and Materials Science, University of California, One Shields Ave, Davis, \\ CA 95616 \\ ** Mathematics Department, University of California, One Shields Ave, Davis, CA 95616 \\ *** Molecular and Cellular Biology Department, University of California, One Shields Ave, Davis, \\ CA 95616 \\ **** Lawrence Livermore National Laboratory, 7000 East Avenue, Livermore, CA 94550
}

Ternary oxides of cubic perovskite structure have electrical properties such as ferro-electricity, mixed conductivity and high- $\mathrm{T}_{\mathrm{c}}$ superconductivity. In perovskite high- $\mathrm{T}_{\mathrm{c}}$ superconductors $\left(\mathrm{YBa}_{2} \mathrm{Cu}_{3} \mathrm{O}_{7}\right)$, grain boundaries $(\mathrm{GB})$ produce a pronounce reduction in the critical current density [1]. In some instances, GBs improve the properties such as conductivity, dielectric constant $\left(\mathrm{BaTiO}_{3}\right.$ and $\left.\mathrm{SrTiO}_{3}\right)$, and magneto-resistance of manganates $\left(\mathrm{La}_{1-\mathrm{x}} \mathrm{Ca}_{\mathrm{x}} \mathrm{MnO}_{3}, \mathrm{SrCaMnO}_{3}\right)$ [2], [3]. GB structure of ternary oxide Strontium Titanate (STO) has the potential of improving the properties of these materials. STO has a large dielectric constant, high dispersion frequency, and small temperature dependence of the dielectric constant. Recently, it is suggested as a possible gate dielectric to replace $\mathrm{SiO}_{2}$ as a gate oxide in field effect transistors. It has a cubic perovskite structure with a lattice constant of $0.3905 \mathrm{~nm}$, which is closely related to a lattice parameter of number of other perovskite oxides, and this makes STO a popular substrate. There are some other applications of STO such as varistors, ferroelectrics, and oxygen sensors [4]. The difference in GB structure from interior of the grains influences the bulk properties and also applications. GB structures of a perovskite should be investigated in details to understand its properties.

To investigate the structure of STO GB, JEOL 2100 equipped with spherical aberration corrector (Cs corrector) is used in scanning transmission electron microscopy (STEM) mode. By using STEM, it is possible to form small and focused electron beam that is essential for spatial resolution of imaging and other microanalysis techniques. Cs corrector improves point-to-point spatial resolution for imaging and for microanalysis. Peak intensities also increase along with total integrated intensity of the incident electron beam. Delocalization of object information which is proportional to Cs is strongly reduced; this is mostly important in high resolution interface imaging [5].

In one of our recent studies, $6^{0}$ tilt GB of STO is investigated by using Cs corrected STEM and electron energy loss spectroscopy (EELS). A variety of dislocation structures along with standard types of dislocation structures are found along the GB. The standard types such as Sr-rich and Tirich dislocation cores have been observed (Fig. 1). Sr-rich core has a double column of Sr atoms in the center of the dislocation. The presence of a double Sr-column is assumed to be a $2 \times 1$ reconstruction of a Sr-single column. Ti-core has a double Ti-O column in the core center. This double column presence is thought to have full Ti site occupancy, however only alternating $\mathrm{O}$ sites are occupied on both columns. Three other most commonly observed dislocation cores are termed as elongated, composite, and transformed. In the new variant elongated core, a splitting of the atomic column along GB is observed immediately adjacent to the typical column doublet seen in the standard core structure (Fig. 2(a)). However, in the composite's dislocation core, (100) lattice plane terminates at two different locations corresponding to the two sublattice plane. Sr column and Ti-O column doublets are observed in this case (Fig. 2(b)). An unusual structure is observed in the 
transformed core where doublets are replaced by two sets of triplets (Fig. 2(c)) [6]. Transformed core seems to have a chemical composition that is different from STO. It appears as a nanotube of TiO embedded in STO matrix. Such variations of dislocation structures on GB influence the bulk properties of materials, enhance the scope of GB engineering (through doping). Further investigations cover the dislocation core in low angle $\left(2^{0}\right.$ tilt $) \mathrm{GB}$.

References

[1] D. M. Feldmann et al., Nature, 414 (2001) 368.

[2] S. P, Isaac et al., Nature, 387 (1997) 266.

[3] W. Heywang, J. Am. Ceram. Soc., 47 (1964) 484.

[4] Z. Zhang et al., Physical Review B 66(2002) 094108.

[5] C. Hetherington, Materials Today, (2004) 50.

[6] J. P. Buban et al., J. Mater. Res., 24 (2009) 2191.

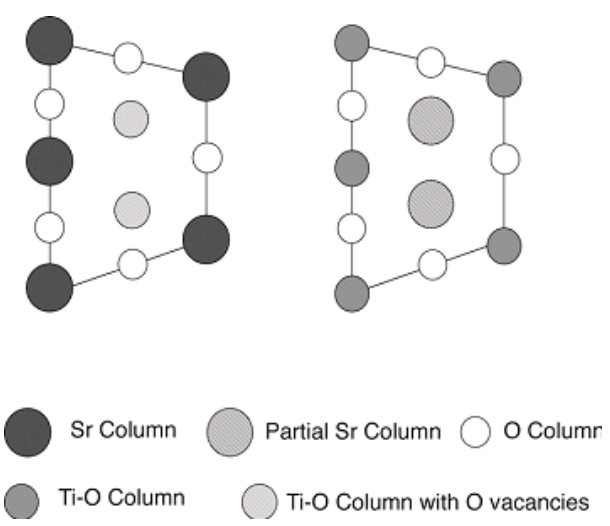

FIG. 1. Standard types edge dislocation [001] cores in STO - Sr-rich (left) and Ti-rich (right).

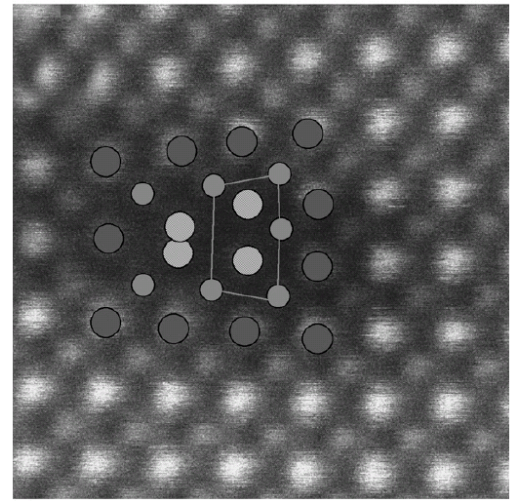

(a)

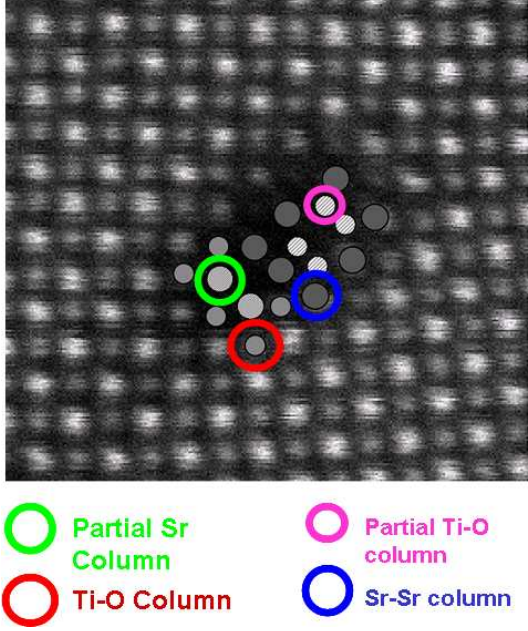

(b)

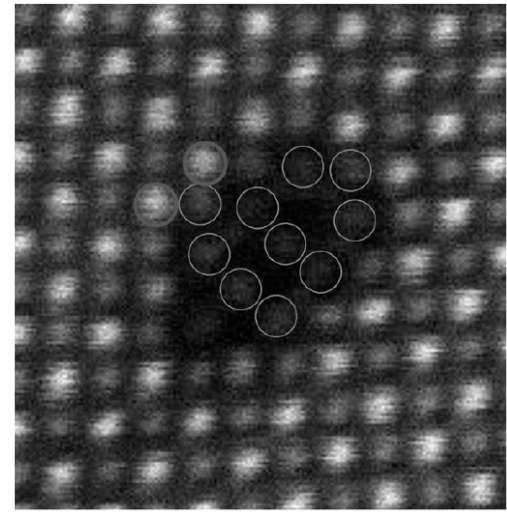

(c)

FIG. 2. STEM image (a) elongated, (b) composite, and (c) transformed dislocation core. 